\title{
Computation of Time Probability Distributions for the Occurrence of Uncertain Future Events
}

\author{
David E. Acuña-Ureta ${ }^{\mathrm{a}, \mathrm{b}, \mathrm{c}, 1, *}$, Marcos E. Orchard ${ }^{\mathrm{a}}$, Patrick Wheeler ${ }^{\mathrm{b}}$ \\ ${ }^{a}$ Department of Electrical Engineering, Faculty of Mathematical and Physical Sciences, \\ Universidad de Chile, Av. Tupper 2007, Santiago, Chile \\ ${ }^{b}$ Power Electronics, Machines and Control Group, University of Nottingham, Nottingham, \\ NG7 2RD, UK \\ ${ }^{c}$ Resilience Engineering Research Group, University of Nottingham, Nottingham, NG7 \\ 2RD, UK
}

\begin{abstract}
The determination of the time at which an event may take place in the future is a well-studied problem in a number of science and engineering disciplines.

Indeed, for more than fifty years, researchers have tried to establish adequate methods to characterize the behaviour of dynamic systems in time and implement predictive decision-making policies. Most of these efforts intend to model the evolution in time of nonlinear dynamic systems in terms of stochastic processes; while defining the occurrence of events in terms of first-passage time problems with thresholds that could be either deterministic or probabilistic in nature. The random variable associated with the occurrence of such events has been determined in closed-form for a variety of specific continuous-time diffusion models, being most of the available literature motivated by physical phenomena. Unfortunately, literature is quite limited in terms of rigorous studies related to discrete-time stochastic processes, despite the tremendous amount of digital information that is currently being collected worldwide. In this regard, this article provides a mathematically rigorous formalization for the problem of computing the probability of occurrence of uncertain future events in both discrete- and
\end{abstract}

\footnotetext{
${ }^{*}$ Corresponding author

Email address: david.acuna@ing.puc.cl (David E. Acuña-Ureta)

${ }^{1}$ Currently with the Department of Mechanical and Metallurgical Engineering and with the Department of Electrical Engineering at Pontificia Universidad Católica de Chile, Av. Vicuña Mackenna 4860, Santiago, Chile.
}

Preprint submitted to Mechanical Systems and Signal Processing

October 7, 2020 
continuous-time stochastic processes, by extending the notion of thresholds in first-passage time problems to a fully probabilistic notion of "uncertain events" and "uncertain hazard zones". We focus on discrete-time applications by showing how to compute those probability measures and validate the proposed framework by comparing to the results obtained with Monte Carlo simulations; all motivated by the problem of fatigue crack growth prognosis.

Keywords: First-hitting time, First-passage time, Time of Failure probability, Remaining useful life, Fatigue crack prognosis

\section{1. Introduction}

One of the motivations behind the use of mathematical models to characterize the evolution in time of dynamic systems is to provide the means to predict and anticipate the occurrence of possibly critical future events. Many different mathematical frameworks can be used for this purpose, and the "best" choice for a model structure will largely depend on the specific application domain. Dynamic models including an explicit characterization of uncertainty sources (e.g., those that include stochastic equations) are particularly suitable to quantify the risk associated with the occurrence of events, since they provide a rigorous mathematical framework for the computation of probability measures. In this context, the conditions that define the occurrence of events have been typically defined in terms of a "threshold", so that the event of interest is always triggered when a scalar function of the system states reaches this threshold for the first time. Naturally, this implies the assumption that the requirements needed to trigger the occurrence of events can always be represented by a deterministic function of the system condition. As the system condition randomly evolves in time (i.e., the condition indicator is a stochastic process), a probability distribution for the threshold first hitting time is therefore induced: the First-Passage Time (FPT) [1] or First-Hitting Time (FHT) [5, 6] probability distribution. On the one hand, this concept is equivalent to duration models [7. 8] and, although understood in a different context, it is also analogous to 
survival probability in statistics 9 11]. On the other hand, in the engineering discipline of Prognostics and Health Management (PHM) these concepts are related to the Remaining Useful Life (RUL), End-of-Life (EoL), Time-of-Failure (ToF) and Time-to-Failure (TtF) probability distributions [12 14].

Efforts on finding analytical expressions for FPT probability distributions have been carried out on many disciplines and application domains such as in chemistry [15, 16], physics [17, 18, biology [19, 20, neurobiology [21, 22, epidemiology [23], psychology 24, finance [25, 26], economy [27, 28], reliability theory [29, 30, among others [1, 2]. Nonetheless, it is important to emphasize the fact that most of these research efforts have focused on continuous-time [31 37, rather than discrete-time systems [27, 38 40] (except the case of autoregressive models [39, 41, 48]). In continuous-time systems, the FPT probability distribution constitutes the solution to particular Stochastic Differential Equation (SDE) with boundary conditions, which is typically solved using transformations [49 51] or on eigenfunction expansions 32, 50 (most of the times numerically approximated). Derivations of direct closed-form expressions are constrained to just a few standard cases related to Brownian motion, like in [52], and some other direct approximations [53 65]. Although it may be natural to think that events occur when some threshold or region is reached by a variable (or condition indicator) that is evolving in time, in some cases it is not straightforward to determine an appropriate value for this threshold. In this regard, and to the best of our knowledge, just a handful of contributions have aimed at incorporating the notion of random thresholds 66-71] (and solely for very specific types of stochastic processes).

\subsection{Failure prognosis in the discipline of Prognostics and Health Management}

Fundamental problems of interest in the modern engineering discipline of PHM are, on the one hand, the implementations of Fault Detection and Diagnostics (FDD) schemes and, on the other hand, the prediction of catastrophic system failures (i.e., failure prognosis). In this regard, it is noteworthy that a clear distinction should be made between the concepts of "faults" (abnor- 
mal conditions in which the systems is still operative) and catastrophic failures (which imply the total inoperatibility of a system), as it is indicated in recent and comprehensive surveys on FDD and failure prognostic approaches [72. The concept of hazard zone 73 arose as an extension to the typical threshold standpoint found in FDD schemes by defining a likelihood over the state-space in regions suggesting faulty conditions. In [74, 75, there was an attempt to provide a more general insight, but it was restricted to Markov processes. Also, there was an underlying hypothesis of statistical independence in the proposed probability measures; although these measures have still proven to be useful to define a functional cost criterion for prognostic algorithm design [76. Moreover, despite the fact that hazard zones are well known and accepted in the PHM community [13], the current state-of-the-art formalization of failure prognosis problem [77, 78, still defines failure events with the classical deterministic threshold approach, is restricted to events over Markov processes, lacks of mathematical demonstrations, and has led to inconsistencies when computing FPT probability distributions with methods different from those simulating complete state trajectories of systems [74].

\subsection{Uncertainty characterization in Reliability-Based Optimization}

The discipline of Reliability Analysis has been a precursor to PHM regarding the study of risks associated with the design and operation of engineering systems. Particularly, the contribution of Reliability-Based Optimization (RBO) 79 82 to this specific aspect is noteworthy. It is only natural, then, to scrutinize how events (or failures) have been defined in the specific context of RBO and the manner in which the concept of uncertainty have been incorporated into these definitions. As it will be shown below, RBO corresponds to a good example for the notion of thresholds-triggered events, even though the value associated with these thresholds might be random in nature and, thus, endowed with probability distributions.

RBO defines an optimization problem for the design of engineering systems under uncertainty. This problem can be formulated in different ways, although 
minimization of expected life time costs, and particularly corrective maintenance costs, is particularly relevant. Indeed, without loss of generality, the RBO problem can be formulated as 81]:

$$
\begin{gathered}
\min _{z \in \Omega_{z}} \mathbb{E}\{C(z, \Phi)\} \\
\text { s.t. } h_{i}(z) \leq 0 \quad, i=1, \ldots, n_{C} \\
P_{j}(z) \leq P_{j}^{t o l}, j=1, \ldots, n_{P},
\end{gathered}
$$

where the objective function is the expectation of a cost $C(\cdot)$, which depends on the vector $z$ (design variables) and a random vector $\Phi$ (uncertain parameters). The functions $h_{i}(\cdot)$ represent constraints to the problem, and $P_{j}(\cdot)$ denotes the probability of occurrence of a $j$-th event. Consequently,

$$
P_{j}(z)=\mathbb{P}\left(g_{j}(z, \Phi) \leq 0\right)=\int_{\left\{\phi \in \Omega_{\phi}: g_{j}(z, \phi) \leq 0\right\}} p(\phi \mid z) d \phi,
$$

where $p(\phi \mid z)$ is the joint probability density function of the random vector of uncertain parameters $\Phi$. The function

$$
g_{j}(z, \Phi)=B_{j}-r_{j}(z, \Phi)
$$

is a performance function associated with the occurrence of a certain event. The function $r_{j}(z, \Phi)$ characterizes the response of the system (i.e., demand), whereas $B_{j}$ corresponds to a -possibly random- threshold of maximum tolerance (i.e., capacity). Therefore, an event in RBO occurs if $g_{j}(z, \Phi) \leq 0$ or, equivalently, if $B_{j} \leq r_{j}(z, \Phi)$. As a result, if $B_{j}$ is a random variable, the probability of occurrence for the $j$-th event is given by:

$$
\begin{aligned}
P_{j}(z) & =\mathbb{P}\left(g_{j}(z, \Phi) \leq 0\right) \\
& =\mathbb{P}\left(B_{j} \leq r_{j}(z, \Phi)\right) \\
& =\int_{b_{j} \in \Omega_{B_{j}}} \mathbb{P}\left(b_{j} \leq r_{j}(z, \Phi) \mid b_{j}\right) p\left(b_{j}\right) d b_{j},
\end{aligned}
$$

where $p\left(b_{j}\right)$ denotes a probability density function associated with a threshold of maximum tolerance $b_{j}$. As Eq. [6] indicates, the occurrence of events in $\mathrm{RBO}$ are triggered by situations in which a threshold is violated, although it is allowed to characterize the values of these thresholds using random variables. 


\subsection{Structure of the article and main contributions}

In this paper we provide a mathematically rigorous formalization for the time of occurrence of uncertain future events, characterized over both discreteand continuous-time stochastic processes by extending the classical deterministic threshold crossing standpoint to a probabilistic notion of uncertain event, equivalent to that of uncertain hazard zone in PHM [73. Particularly, Section 2 presents explicit semi-closed expressions for the associated probability measures, which are derived and demonstrated using Probability Theory. In addition to these theoretical contributions, in Section 3 we present a friendly explanation of the practical implications related to these theoretical concepts, using for this purpose a case study based on the problem of crack growth prognostics (discrete-time). Results obtained using the proposed semi-closed expressions for the probability of failure are validated and compared with those obtained by using Monte Carlo simulations. Finally, in Section 4 we summarize the main conclusions of this research effort.

\section{Occurrence Probability of Uncertain Future Events}

Let us consider a probability space $(\Omega, \mathcal{F}, \mathbb{P})$ and a measurable space $(\mathbb{X}, \Sigma)$. Also, let $X: \mathbb{T} \cup\{0\} \times \Omega \rightarrow \mathbb{X}, \mathbb{T} \in\left\{\mathbb{N}, \mathbb{R}_{+}\right\}$, be a stochastic process; and $F_{X_{\tau}}$ denote the respective probability measure in $(\mathbb{X}, \Sigma)$ induced by $X_{\tau}, \tau \in \mathbb{T}$.

Definition 1. [Uncertain Event Process $\mathcal{E}$ Likelihood] Let $\mathcal{E}$ denote an event of interest. An uncertain event process is defined as a function $E$ : $\mathbb{T} \times \mathbb{X} \rightarrow\left\{\mathcal{E}, \mathcal{E}^{c}\right\}$ such that

$$
\begin{aligned}
\mathbb{P}\left(E_{\tau}=\mathcal{E}\right) & =\int_{\Omega} \mathbb{P}\left(E_{\tau}=\mathcal{E}, X_{\tau}(\omega)\right) d \mathbb{P}(\omega) \\
& =\int_{\mathbb{X}_{\tau}} \mathbb{P}\left(E_{\tau}=\mathcal{E} \mid x_{\tau}\right) d F_{X_{\tau}}\left(x_{\tau}\right), \forall \tau \in \mathbb{T} .
\end{aligned}
$$

Additionally, we define an uncertain event likelihood function as $\mathbb{P}\left(E_{\tau}=\right.$ $\mathcal{E} \mid x): \mathbb{T} \times \mathbb{X} \rightarrow[0,1]$ 
Thus, an event process $\left\{E_{\tau}\right\}_{\tau \in \mathbb{T}}$ describes a random variable evolving in time associated with the occurrence of an uncertain event whose statistics are subjected only to those of a stochastic process $\left\{X_{\tau}\right\}_{\tau \in \mathbb{T} \cup\{0\}}$ evaluated at the same time instant. Indeed, given $i, j \in \mathbb{T}, i \neq j$, we have

$$
\mathbb{P}\left(\left\{E_{i}=\mathcal{E}\right\},\left\{E_{j}=\mathcal{E}\right\} \mid\left\{X_{\tau}\right\}_{\tau \in \mathbb{T}}\right)=\mathbb{P}\left(E_{i}=\mathcal{E} \mid X_{i}\right) \mathbb{P}\left(E_{j}=\mathcal{E} \mid X_{j}\right) .
$$

In general, $E_{\tau}$ is independent of any other variable as long as it is conditioned on $X_{\tau}, \forall \tau \in \mathbb{T}$. This is a quite important property that will be used later.

Remark 1. [Time-variant definition of uncertain events] The time index $\tau$ of the binary random variable $E_{\tau}$, in the definition of uncertain event likelihood functions (see Definition 11), denotes a time dependence associated with the concept of uncertain event. For this reason, the definition of the probability $\mathbb{P}\left(E_{\tau}=\mathcal{E} \mid x\right)$ uses " $x$ " as conditioning argument instead of " $x_{\tau}$ "; i.e., the system state " $x$ " solely corresponds to an argument in the time-dependant likelihood function. In other words, the time dependency of the uncertain event likelihood function determines the manner in which the definition of the event of interest changes over time (see Section 3.2 for an example of a time-invariant definition of uncertain event likelihood function).

Remark 2. [Particular case: Threshold] According to Definition 1, determining $\mathbb{P}\left(E_{\tau}=\mathcal{E} \mid x\right)$ as a function of $x \in \mathbb{X}$ corresponds exactly to a probabilistic description of the occurrence of an uncertain event $\mathcal{E}$. By assuming $\mathbb{X}=\mathbb{R}$, for example, and defining $\mathbb{P}\left(E_{\tau}=\mathcal{E} \mid x\right)=\mathbb{1}_{\mathbb{X}_{\mathcal{E}}}(x)$, with $\mathbb{X}_{\mathcal{E}}=\{x \in \mathbb{R}: x>c, c \in \mathbb{R}\}$, we get the classical threshold crossing event setting studied so far in the literature, where $E_{\tau}(x)$ conditional to a fixed $x \in \mathbb{R}$ is no longer a random variable:

$$
E_{\tau}(x)=\left\{\begin{array}{l}
\mathcal{E}, x \in \mathbb{X}_{\mathcal{E}} \\
\mathcal{E}^{c}, \sim .
\end{array}\right.
$$

Remark 3. [Hazard zone] The uncertain event likelihood function $\mathbb{P}\left(E_{\tau}=\right.$ $\mathcal{E} \mid x)$ as a function of $x \in \mathbb{X}$ is exactly what is understood as hazard zone [73] in the discipline of PHM. 
Now, let us introduce a formal definition for the first time of occurrence of an uncertain event.

Definition 2. [First Event Time] Let $\left\{X_{\tau}\right\}_{\tau \in \mathbb{T} \cup\{0\}}$ be a stochastic process and $\left\{E_{\tau}\right\}_{\tau \in \mathbb{T}}$ be an event process, respectively. The first time of occurrence of an event $\mathcal{E}$ after a time instant $\tau_{p} \in \mathbb{T} \cup\{0\}$ is defined as

$$
\tau_{\mathcal{E}}\left(\tau_{p}\right):=\inf \left\{\tau \in \mathbb{T}:\left\{\tau>\tau_{p}\right\} \wedge\left\{E_{\tau}=\mathcal{E}\right\}\right\} .
$$

With these few definitions, the probability distribution associated to $\tau_{\mathcal{E}}$ can be mathematically formalized in a general way for both discrete- and continuoustime stochastic processes as follows.

\subsection{Discrete-Time Stochastic Processes}

Let $\left\{X_{k}\right\}_{k \in \mathbb{N} \cup\{0\}}$ be a stochastic process and $\left\{E_{k}\right\}_{k \in \mathbb{N}}$ be an uncertain event process. The probability mass function associated to $\tau_{\mathcal{E}}=\tau_{\mathcal{E}}\left(k_{p}\right), k_{p} \in \mathbb{N} \cup$ $\{0\}$, can be obtained using an expression with the same structure of survival probability, as shown below:

$$
\begin{aligned}
& \mathbb{P}\left(\tau_{\mathcal{E}}=k\right):=\mathbb{P}\left(\left\{E_{k}=\mathcal{E}\right\},\left\{E_{j}=\mathcal{E}^{c}\right\}_{j=k_{p}}^{k-1}\right) \\
& \quad=\mathbb{P}\left(E_{k}=\mathcal{E} \mid\left\{E_{j}=\mathcal{E}^{c}\right\}_{j=k_{p}}^{k-1}\right) \mathbb{P}\left(\left\{E_{j}=\mathcal{E}^{c}\right\}_{j=k_{p}}^{k-1}\right) \\
& \quad \vdots \\
& \quad=\mathbb{P}\left(E_{k}=\mathcal{E} \mid\left\{E_{j}=\mathcal{E}^{c}\right\}_{j=k_{p}}^{k-1}\right) \prod_{j=k_{p}+1}^{k-1} \mathbb{P}\left(E_{j}=\mathcal{E}^{c} \mid\left\{E_{i}=\mathcal{E}^{c}\right\}_{i=k_{p}}^{j-1}\right){\mathbb{P}\left(E_{k_{p}}=\mathcal{E}^{c}\right)^{1}}^{1} \\
& \quad=\mathbb{P}\left(E_{k}=\mathcal{E} \mid \tau_{\mathcal{E}} \geq k\right) \prod_{j=k_{p}+1}^{k-1} \mathbb{P}\left(E_{j}=\mathcal{E}^{c} \mid \tau_{\mathcal{E}} \geq j\right)
\end{aligned}
$$

Alternatively, there is a recursive way to express $\mathbb{P}\left(\tau_{\mathcal{E}}=k\right)$ according to [27, which is developed below but under the generalized notion of uncertain event presented in Definition 1 If an event occurs at time $k$, it implies that $k_{p}<\tau_{\mathcal{E}} \leq k$, and by the Law of Total Probability it can be obtained

$$
\mathbb{P}\left(E_{k}=\mathcal{E}\right)=\sum_{j=k_{p}+1}^{k} \mathbb{P}\left(E_{k}=\mathcal{E} \mid \tau_{\mathcal{E}}=j\right) \mathbb{P}\left(\tau_{\mathcal{E}}=j\right)
$$




$$
=\mathbb{P}\left(E_{k}=\mathcal{E} \mid \tau_{\mathcal{E}}=k\right) \mathbb{P}\left(\tau_{\mathcal{E}}=k\right)+\sum_{j=k_{p}+1}^{k-1} \mathbb{P}\left(E_{k}=\mathcal{E} \mid \tau_{\mathcal{E}}=j\right) \mathbb{P}\left(\tau_{\mathcal{E}}=j\right)
$$

Thus, provided $\mathbb{P}\left(E_{k}=\mathcal{E} \mid \tau_{\mathcal{E}}=k\right)=1$, it yields

$$
\mathbb{P}\left(\tau_{\mathcal{E}}=k\right)=\mathbb{P}\left(E_{k}=\mathcal{E}\right)-\sum_{j=k_{p}+1}^{k-1} \mathbb{P}\left(E_{k}=\mathcal{E} \mid \tau_{\mathcal{E}}=j\right) \mathbb{P}\left(\tau_{\mathcal{E}}=j\right)
$$

Proof.

Using Eq. 20, Eq. (19) can be obtained as shown below

$$
\begin{aligned}
\mathbb{P}\left(\tau_{\mathcal{E}}=k\right) & =\mathbb{P}\left(E_{k}=\mathcal{E}\right)-\sum_{j=k_{p}+1}^{k-1} \mathbb{P}\left(E_{k}=\mathcal{E} \mid \tau_{\mathcal{E}}=j\right) \mathbb{P}\left(\tau_{\mathcal{E}}=j\right) \\
& =\mathbb{P}\left(E_{k}=\mathcal{E}\right)-\sum_{j=k_{p}+1}^{k-1} \mathbb{P}\left(\tau_{\mathcal{E}}=j \mid E_{k}=\mathcal{E}\right) \mathbb{P}\left(E_{k}=\mathcal{E}\right) \\
& =\mathbb{P}\left(E_{k}=\mathcal{E}\right)\left(1-\sum_{j=k_{p}+1}^{k-1} \mathbb{P}\left(\tau_{\mathcal{E}}=j \mid E_{k}=\mathcal{E}\right)\right) \\
& =\mathbb{P}\left(E_{k}=\mathcal{E}\right)\left(1-\mathbb{P}\left(\tau_{\mathcal{E}}<k \mid E_{k}=\mathcal{E}\right)\right) \\
& =\mathbb{P}\left(E_{k}=\mathcal{E}\right) \mathbb{P}\left(\tau_{\mathcal{E}} \geq k \mid E_{k}=\mathcal{E}\right) \\
& =\mathbb{P}\left(E_{k}=\mathcal{E} \mid \tau_{\mathcal{E}} \geq k\right) \mathbb{P}\left(\tau_{\mathcal{E}} \geq k\right)
\end{aligned}
$$


However, note that

$$
\begin{aligned}
\mathbb{P}\left(\tau_{\mathcal{E}} \geq k\right) & =1-\mathbb{P}\left(\tau_{\mathcal{E}}<k\right) \\
& =1-\mathbb{P}\left(\tau_{\mathcal{E}}<k-1\right)-\mathbb{P}\left(\tau_{\mathcal{E}}=k-1\right) \\
& =\mathbb{P}\left(\tau_{\mathcal{E}} \geq k-1\right)-\mathbb{P}\left(\tau_{\mathcal{E}}=k-1\right) \\
& =\mathbb{P}\left(\left\{E_{j}=\mathcal{E}^{c}\right\}_{j=k_{p}}^{k-2}\right)-\mathbb{P}\left(\left\{E_{k-1}=\mathcal{E}\right\},\left\{E_{j}=\mathcal{E}^{c}\right\}_{j=k_{p}}^{k-2}\right) \\
& =\mathbb{P}\left(\left\{E_{j}=\mathcal{E}^{c}\right\}_{j=k_{p}}^{k-2}\right)-\mathbb{P}\left(E_{k-1}=\mathcal{E} \mid\left\{E_{j}=\mathcal{E}^{c}\right\}_{j=k_{p}}^{k-2}\right) \mathbb{P}\left(\left\{E_{j}=\mathcal{E}^{c}\right\}_{j=k_{p}}^{k-2}\right) \\
& =\mathbb{P}\left(\left\{E_{j}=\mathcal{E}^{c}\right\}_{j=k_{p}}^{k-2}\right)\left(1-\mathbb{P}\left(E_{k-1}=\mathcal{E} \mid\left\{E_{j}=\mathcal{E}^{c}\right\}_{j=k_{p}}^{k-2}\right)\right) \\
& =\mathbb{P}\left(\tau_{\mathcal{E}} \geq k-1\right) \mathbb{P}\left(E_{k-1}=\mathcal{E}^{c} \mid \tau_{\mathcal{E}} \geq k-1\right)
\end{aligned}
$$

By iterating this result, it yields

$$
\mathbb{P}\left(\tau_{\mathcal{E}} \geq k\right)=\prod_{j=k_{p}+1}^{k-1} \mathbb{P}\left(E_{j}=\mathcal{E}^{c} \mid \tau_{\mathcal{E}} \geq j\right)
$$

Before presenting the previous results in a formal theorem, please note the following. Since each $E_{k}$ depends on $X_{k}$, using the property illustrated with Eq. (9) and the concept of uncertain event likelihood function introduced in Definition 1 , it can be obtained:

$$
\begin{aligned}
& \mathbb{P}\left(\tau_{\mathcal{E}}=k\right)=\mathbb{P}\left(E_{k}=\mathcal{E} \mid \tau_{\mathcal{E}} \geq k\right) \prod_{j=k_{p}+1}^{k-1} \mathbb{P}\left(E_{j}=\mathcal{E}^{c} \mid \tau_{\mathcal{E}} \geq j\right) \\
& =\int_{\mathbb{X}_{k_{p}+1: k}} \mathbb{P}\left(E_{k}=\mathcal{E} \mid\left\{\tau_{\mathcal{E}} \geq k\right\}, x_{k_{p}+1: k}\right) \prod_{j=k_{p}+1}^{k-1} \mathbb{P}\left(E_{j}=\mathcal{E}^{c} \mid\left\{\tau_{\mathcal{E}} \geq j\right\}, x_{k_{p}+1: k}\right) d F_{X_{k_{p}+1: k}}\left(x_{k_{p}+1: k}\right) \\
& =\int_{\mathbb{X}_{k_{p}+1: k}} \mathbb{P}\left(E_{k}=\mathcal{E} \mid x_{k}\right) \prod_{j=k_{p}+1}^{k-1} \mathbb{P}\left(E_{j}=\mathcal{E}^{c} \mid x_{j}\right) d F_{X_{k_{p}+1: k}}\left(x_{k_{p}+1: k}\right) \\
& =\int_{\mathbb{X}_{k_{p}+1: k}} \mathbb{P}\left(E_{k}=\mathcal{E} \mid x_{k}\right) \prod_{j=k_{p}+1}^{k-1}\left(1-\mathbb{P}\left(E_{j}=\mathcal{E} \mid x_{j}\right)\right) d F_{X_{k_{p}+1: k}}\left(x_{k_{p}+1: k}\right)
\end{aligned}
$$

This expression is used later in Section 3.2 to implement a procedure to compute these probabilities based on numeric methods. 
Theorem 1. [First Event Time in Stochastic Processes] Let $\left\{X_{k}\right\}_{k \in \mathbb{N} \cup\{0\}}$ be a stochastic process and $\left\{E_{k}\right\}_{k \in \mathbb{N}}$ be an uncertain event process, respectively. If the first time of occurrence of the event $\mathcal{E}, \tau_{\mathcal{E}}=\tau_{\mathcal{E}}\left(k_{p}\right)$, with $k_{p} \in \mathbb{N} \cup\{0\}$, is such that $\tau_{\mathcal{E}}<+\infty, \mathbb{P}-$ a.s., then the mapping $\mathbb{P}\left(\tau_{\mathcal{E}}=\cdot\right): \mathbb{N} \rightarrow[0,1]$ exists and is well-defined in terms of its uncertain event likelihood function as:

$\mathbb{P}\left(\tau_{\mathcal{E}}=k\right):=\int_{\mathbb{X}_{k_{p}+1: k}} \mathbb{P}\left(E_{k}=\mathcal{E} \mid x_{k}\right) \prod_{j=k_{p}+1}^{k-1}\left(1-\mathbb{P}\left(E_{j}=\mathcal{E} \mid x_{j}\right)\right) d F_{X_{k_{p}+1: k}}\left(x_{k_{p}+1: k}\right)$.

Therefore,

$$
\mathbb{P}_{\mathcal{E}}(A)=\sum_{k \in A} \mathbb{P}\left(\tau_{\mathcal{E}}=k\right), \forall A \in 2^{\mathbb{N}},
$$

2) By definition, because

$$
0 \leq \mathbb{P}\left(\tau_{\mathcal{E}}=k\right), \forall k \in \mathbb{N} \Rightarrow 0 \leq \mathbb{P}_{\mathcal{E}}(A), \forall A \in 2^{\mathbb{N}},
$$


and, on the other hand,

$$
\begin{aligned}
A \subseteq \mathbb{N} & \Rightarrow \sum_{k \in A} \mathbb{P}\left(\tau_{\mathcal{E}}=k\right) \leq \sum_{k \in \mathbb{N}} \mathbb{P}\left(\tau_{\mathcal{E}}=k\right) \\
& \Leftrightarrow \mathbb{P}_{\mathcal{E}}(A) \leq \mathbb{P}_{\mathcal{E}}(\mathbb{N})=1, \forall A \in 2^{\mathbb{N}} .
\end{aligned}
$$

3) Let $\left\{A_{k} \in 2^{\mathbb{N}}\right\}_{k \in \mathbb{N}}$ such that $A_{i} \cap A_{j}=\phi, \forall i \neq j$. By definition,

$$
\begin{aligned}
\mathbb{P}_{\mathcal{E}}\left(\cup_{k \in \mathbb{N}} A_{k}\right) & =\sum_{j \in \cup_{k \in \mathbb{N}} A_{k}} \mathbb{P}\left(\tau_{\mathcal{E}}=j\right) \\
& =\sum_{k \in \mathbb{N}} \sum_{j \in A_{k}} \mathbb{P}\left(\tau_{\mathcal{E}}=j\right) \\
& =\sum_{k \in \mathbb{N}} \mathbb{P}_{\mathcal{E}}\left(A_{k}\right)
\end{aligned}
$$

140

\subsection{Continuous-Time Stochastic Processes}

Let $\left\{X_{t}\right\}_{t \in \mathbb{R}_{+} \cup\{0\}}$ be a stochastic process and $\left\{E_{t}\right\}_{t \in \mathbb{R}_{+}}$be an uncertain event process. By definition, if there was a probability density function associated to $\tau_{\mathcal{E}}=\tau_{\mathcal{E}}\left(t_{p}\right), t_{p} \in \mathbb{R}_{+} \cup\{0\}$, then it could be obtained using an expression with the same structure of survival probability, as shown below:

$$
\begin{aligned}
p\left(\tau_{\mathcal{E}}=t\right) & :=\mathbb{P}\left(\left\{E_{t}=\mathcal{E}\right\},\left\{E_{\tau}=\mathcal{E}^{c}\right\}_{\tau \in\left(t_{p}, t\right)}\right) \\
& =\mathbb{P}\left(\left\{E_{t}=\mathcal{E}\right\},\left\{\tau_{\mathcal{E}} \geq t\right\}\right) \\
& =\mathbb{P}\left(E_{t}=\mathcal{E} \mid \tau_{\mathcal{E}} \geq t\right) \mathbb{P}\left(\tau_{\mathcal{E}} \geq t\right)
\end{aligned}
$$

with $\mathbb{P}\left(\tau_{\mathcal{E}} \geq t\right)=1-\mathbb{P}\left(\tau_{\mathcal{E}}<t\right)$. Let $\mathcal{B}\left(\mathbb{R}_{+}\right)$and $\lambda=\lambda\left(\mathbb{R}_{+}\right)$denote the Borel $\sigma$-algebra and Lebesgue measure in $\mathbb{R}_{+}$, respectively. Let also $F_{\tau_{\mathcal{E}}}(t)=\mathbb{P}\left(\tau_{\mathcal{E}} \leq\right.$ $t$ ) be a probability measure in the measurable space $\left(\mathbb{R}_{+}, \mathcal{B}\left(\mathbb{R}_{+}\right)\right)$such that $F_{\tau_{\mathcal{E}}}<<\lambda$. According to the Theorem of Radon-Nikodym, there is a probability density function $p\left(\tau_{\mathcal{E}}=t\right):=\frac{d F_{\tau_{\mathcal{E}}}}{d \lambda}(t), t \in \mathbb{R}_{+}$, such that

$$
\begin{aligned}
\mathbb{P}\left(\tau_{\mathcal{E}}<t\right) & =\int_{\left(t_{p}, t\right)} d F_{\tau_{\mathcal{E}}}(\tau) \\
& =\int_{\left(t_{p}, t\right)} p\left(\tau_{\mathcal{E}}=\tau\right) d \tau
\end{aligned}
$$




$$
=\int_{\left(t_{p}, t\right)} \mathbb{P}\left(E_{\tau}=\mathcal{E} \mid \tau_{\mathcal{E}} \geq \tau\right) \mathbb{P}\left(\tau_{\mathcal{E}} \geq \tau\right) d \tau
$$

Due to the existence of the aforementioned probability density function, $\mathbb{P}\left(\tau_{\mathcal{E}} \geq t\right)$ must be differentiable

$$
\Rightarrow \frac{d}{d t} \mathbb{P}\left(\tau_{\mathcal{E}} \geq t\right)=-\mathbb{P}\left(E_{t}=\mathcal{E} \mid \tau_{\mathcal{E}} \geq t\right) \mathbb{P}\left(\tau_{\mathcal{E}} \geq t\right),
$$

because $\mathbb{P}\left(E_{t_{p}}=\mathcal{E} \mid \tau_{\mathcal{E}} \geq t_{p}\right)=\mathbb{P}\left(E_{t_{p}}=\mathcal{E}\right)=0$ (at the beginning it was stated that $\tau_{\mathcal{E}}>t_{p}$ ). Integrating over time,

$$
\begin{aligned}
-\int_{\left(t_{p}, t\right)} \mathbb{P}\left(E_{\tau}=\mathcal{E} \mid \tau_{\mathcal{E}} \geq \tau\right) d \tau & =\int_{\left(t_{p}, t\right)} \frac{1}{\mathbb{P}\left(\tau_{\mathcal{E}} \geq \tau\right)} \frac{d}{d \tau} \mathbb{P}\left(\tau_{\mathcal{E}} \geq \tau\right) d \tau \\
& =\int_{\left(t_{p}, t\right)} \frac{d}{d \tau} \log \mathbb{P}\left(\tau_{\mathcal{E}} \geq \tau\right) d \tau \\
& =\log \mathbb{P}\left(\tau_{\mathcal{E}} \geq t\right)-\underset{\log \mathbb{P}\left(\tau_{\mathcal{E}} \geq t_{p}\right)}{0}
\end{aligned}
$$

Thus,

$$
\Rightarrow \mathbb{P}\left(\tau_{\mathcal{E}} \geq t\right)=e^{-\int_{t_{p}}^{t} \mathbb{P}\left(E_{\tau}=\mathcal{E} \mid \tau_{\mathcal{E}} \geq \tau\right) d \tau} .
$$

Before presenting the previous results in a formal theorem, please note the following. Since each $E_{t}$ depends on $X_{t}$, using the property illustrated with Eq. (9) and the concept of uncertain event likelihood function introduced in Definition 1, it can be obtained:

$$
\begin{aligned}
& p\left(\tau_{\mathcal{E}}=t\right)=\mathbb{P}\left(E_{t}=\mathcal{E} \mid \tau_{\mathcal{E}} \geq t\right) e^{-\int_{t_{p}}^{t} \mathbb{P}\left(E_{\tau}=\mathcal{E} \mid \tau_{\mathcal{E}} \geq \tau\right) d \tau} \\
& =\int_{\mathbb{X}_{\left(t_{p}, t\right]}} \mathbb{P}\left(E_{t}=\mathcal{E} \mid\left\{\tau_{\mathcal{E}} \geq t\right\}, x_{\left(t_{p}, t\right]}\right) e^{-\int_{t_{p}}^{t} \mathbb{P}\left(E_{\tau}=\mathcal{E} \mid\left\{\tau_{\mathcal{E}} \geq \tau\right\}, x_{\left(t_{p}, t\right]}\right) d \tau} d F_{\mathbb{X}_{\left(t_{p}, t\right]}}\left(x_{\left(t_{p}, t\right]}\right) \\
& =\int_{\mathbb{X}_{\left(t_{p}, t\right]}} \mathbb{P}\left(E_{t}=\mathcal{E} \mid x_{t}\right) e^{-\int_{t_{p}}^{t} \mathbb{P}\left(E_{\tau}=\mathcal{E} \mid x_{\tau}\right) d \tau} d F_{\mathbb{X}_{\left(t_{p}, t\right]}}\left(x_{\left(t_{p}, t\right]}\right)
\end{aligned}
$$

Theorem 2. [First Event Time in Stochastic Processes] Let $\mathcal{B}\left(\mathbb{R}_{+}\right)$and $\lambda=\lambda\left(\mathbb{R}_{+}\right)$denote the Borel $\sigma$-algebra and Lebesgue measure in $\mathbb{R}_{+}$, respectively. Let also $\left\{X_{t}\right\}_{t \in \mathbb{R}_{+} \cup\{0\}}$ be a stochastic process and $\left\{E_{t}\right\}_{t \in \mathbb{R}_{+}}$be an uncertain event process. If the first time of occurrence of the event $\mathcal{E}, \tau_{\mathcal{E}}=\tau_{\mathcal{E}}\left(t_{p}\right)$, with 
$t_{p} \in \mathbb{R}_{+} \cup\{0\}$, is such that $\tau_{\mathcal{E}}<+\infty, \mathbb{P}-$ a.s., and $F_{\tau_{\mathcal{E}}}<<\lambda$, then the mapping $p\left(\tau_{\mathcal{E}}=\cdot\right): \mathbb{R}_{+} \rightarrow[0,1]$ exists and is well-defined in terms of its uncertain event likelihood function as

$$
\mathbb{P}\left(\tau_{\mathcal{E}}=t\right):=\int_{\mathbb{X}_{\left(t_{p}, t\right]}} \mathbb{P}\left(E_{t}=\mathcal{E} \mid x_{t}\right) e^{-\int_{t_{p}}^{t} \mathbb{P}\left(E_{\tau}=\mathcal{E} \mid x_{\tau}\right) d \tau} d F_{\mathbb{X}_{\left(t_{p}, t\right]}}\left(x_{\left(t_{p}, t\right]}\right) .
$$

Therefore,

$$
\mathbb{P}_{\mathcal{E}}(B)=\int_{B} d F_{\tau_{\mathcal{E}}}(\tau)=\int_{B} p\left(\tau_{\mathcal{E}}=\tau\right) d \tau, \forall B \in \mathcal{B}\left(\mathbb{R}_{+}\right),
$$

2) By definition, because

$$
0 \leq \mathbb{P}\left(\tau_{\mathcal{E}}=t\right), \forall t \in \mathbb{R}_{+} \Rightarrow 0 \leq \mathbb{P}_{\mathcal{E}}(B), \forall B \in \mathcal{B}\left(\mathbb{R}_{+}\right)
$$

and, on the other hand,

$$
\begin{aligned}
B \subseteq \mathbb{R}_{+} & \Rightarrow \int_{B} p\left(\tau_{\mathcal{E}}=\tau\right) d \tau \leq \int_{\mathbb{R}_{+}} p\left(\tau_{\mathcal{E}}=\tau\right) d \tau \\
& \Leftrightarrow \mathbb{P}_{\mathcal{E}}(B) \leq \mathbb{P}_{\mathcal{E}}\left(\mathbb{R}_{+}\right)=1, \quad \forall B \in \mathcal{B}\left(\mathbb{R}_{+}\right) .
\end{aligned}
$$


3) Let $\left\{B_{k} \in \mathcal{B}\left(\mathbb{R}_{+}\right)\right\}_{k \in \mathbb{N}}$ such that $B_{i} \cap B_{j}=\phi, \forall i \neq j$. By definition,

$$
\begin{aligned}
\mathbb{P}_{\mathcal{E}}\left(\cup_{k \in \mathbb{N}} B_{k}\right) & =\int_{\cup_{k \in \mathbb{N}} B_{k}} p\left(\tau_{\mathcal{E}}=\tau\right) d \tau \\
& =\sum_{k \in \mathbb{N}} \int_{B_{k}} p\left(\tau_{\mathcal{E}}=\tau\right) d \tau \\
& =\sum_{k \in \mathbb{N}} \mathbb{P}_{\mathcal{E}}\left(B_{k}\right)
\end{aligned}
$$

151

\section{Uncertain Event Prognosis in Practice}

This section aims at facilitating the transition between theory and practice by providing a friendly interpretation of theorems that constitute the true contribution of this article. In addition, a case study inspired on the problem of fatigue crack growth is used to illustrate the application of these concepts in failure prognostics.

\subsection{A Friendly Interpretation of Theorems 1 and 2}

Since theoretical implications of Theorems 1 and 2 are completely analogous, we will focus on providing adequate interpretations for Theorem 1, which characterizes the probability of future events in discrete-time dynamic systems.

For this purpose, let us assume that it is intended to perform critical event prognostics at a time $k_{p}$. The first element to consider is a proper representation for the dynamic system that characterizes the future evolution of the condition indicator of interest. This representation corresponds to the stochastic process $\left\{X_{k}\right\}_{k \in \mathbb{N} \cup\{0\}}$, where the variable $k$ is a time index (seconds, minutes, hours, cycles of operation) that takes values in the set of natural numbers. System dynamics in typical real-world applications are commonly characterised using Markov processes (i.e. future is independent of the past, conditional on the present), which leads to the state-space model:

$$
x_{k+1}=f\left(x_{k}, u_{k}, \omega_{k}\right),
$$


where $u_{k}$ denotes an exogenous system input and $\omega_{k}$ is a random vector that accounts for model uncertainty, a.k.a. the process noise. It is important to note, however, that the Markovian assumption was used in this section solely for illustrative purposes since, as can be verified in Theorems 1 and 2 , there are no restrictions on the stochastic process that could be used to describe the dynamics of the system.

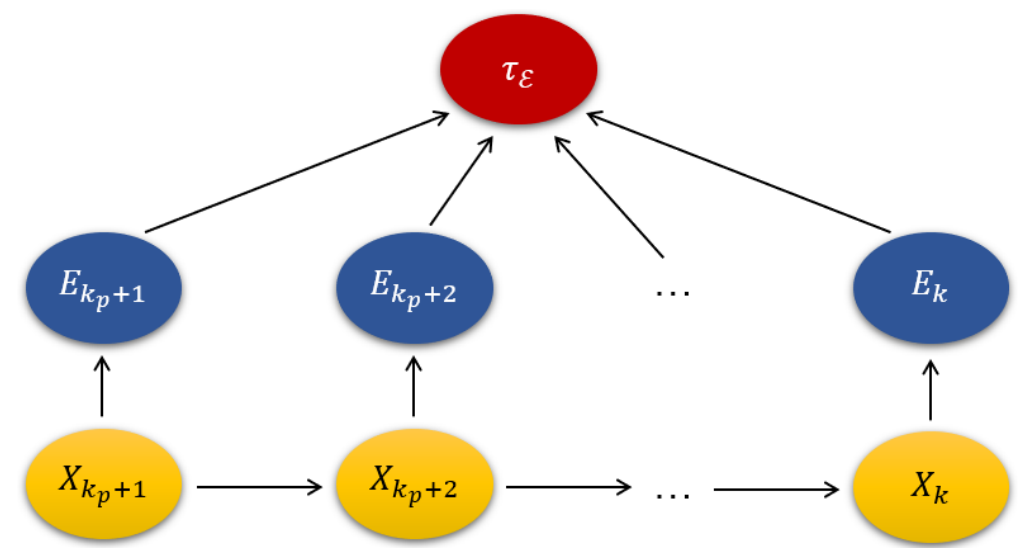

Figure 1: Illustration of statistical dependency relationships in the dynamic system described by Eq. 74. The statistics of $\tau_{\mathcal{E}}$ depend on $\left\{E_{k}\right\}_{k>k_{p}}$, and this dependency is clearly expressed in Eq. 12 (or Eq. 50 in the case of continuous-time systems).

The next step that is required to use Theorem 1 is to characterize the uncertain event process $\left\{E_{k}\right\}_{k \in \mathbb{N}}$. Naturally, this characterization depends on which event $\mathcal{E}$ is sought to be prognosticated. As it is illustrated in Fig. 1, at each future time instant $k, k>k_{p}$, the binary random variable $E_{k}$ indicates whether the event $\mathcal{E}$ has occurred or not, solely depending on the system condition indicator $X_{k}$. This is one of the main concerns that should be settled by the designer of the prognostic algorithm: How can the dependency of $E_{k}$ on $X_{k}$ be determined? What does $\mathcal{E}$ has to do with this dependency? It is important to remark that the event $\mathcal{E}$ must be, in the first place, qualitatively defined; for example:

$$
\mathcal{E}=\text { "Critical system failure". }
$$

The latter would imply that the occurrence of the $\mathcal{E}$ at a time $k$ corresponds 
to a binary random variable $E_{k}$. This variable, in contrast to $\mathcal{E}$, must be defined in terms of a quantitative description given by an uncertain event likelihood function $\mathbb{P}\left(E_{k}=\mathcal{E} \mid x\right)$ for each $k>k_{p}$. Following the aforementioned example, if the system state were to be a one-dimensional fault indicator (scalar value), "Critical system failure" might be declared once the system state reaches an upper threshold $\bar{x}$. With this definition, we have

$$
\mathbb{P}\left(E_{k}=\mathcal{E} \mid x\right)=\mathbb{P}\left(E_{k}=\text { "Critical system failure" } \mid x\right)=\left\{\begin{array}{l}
1, x \geq \bar{x} \\
0, \sim .
\end{array}\right.
$$

Now, the final aim of the prognostic algorithm is to characterize the probability distribution of the random variable $\tau_{\mathcal{E}}$, which denotes the first occurrence time of the event $\mathcal{E}$ in the future. Having defined the uncertain event likelihood function $\mathbb{P}\left(E_{k}=\mathcal{E} \mid x\right)$ for each $k>k_{p}$, it is straightforward to apply Eq. (39) of Theorem 1 (or Eq. 63) of Theorem 2 in the continuous time case) to probabilistically characterize $\tau_{\mathcal{E}}$ :

$$
\mathbb{P}\left(\tau_{\mathcal{E}}=k\right)=\int_{\mathbb{X}_{k_{p}+1: k}} \mathbb{P}\left(E_{k}=\mathcal{E} \mid x_{k}\right) \prod_{j=k_{p}+1}^{k-1}\left(1-\mathbb{P}\left(E_{j}=\mathcal{E} \mid x_{j}\right)\right) d F_{X_{k_{p}+1: k}}\left(x_{k_{p}+1: k}\right)
$$

This expression, however, can often be rewritten in terms the probability density $p\left(x_{k_{p}+1: k}\right)$ as:

$$
\mathbb{P}\left(\tau_{\mathcal{E}}=k\right)=\int_{\mathbb{X}_{k_{p}+1: k}} \mathbb{P}\left(E_{k}=\mathcal{E} \mid x_{k}\right) \prod_{j=k_{p}+1}^{k-1}\left(1-\mathbb{P}\left(E_{j}=\mathcal{E} \mid x_{j}\right)\right) p\left(x_{k_{p}+1: k}\right) d x_{k_{p}+1: k}
$$

The use of the infinitesimal term " $d F_{X_{k_{p}+1: k}}\left(x_{k_{p}+1: k}\right)$ " is a mathematical technicality due to the use of Lebesgue integration, since it is possible to rewrite 
$\mathbb{P}\left(\tau_{\mathcal{E}}=k\right)$ in terms of Riemann integration, except when the probability density $p\left(x_{k_{p}+1: k}\right)$ does not exist, which is rather rare in practice.

Finally, it is noteworthy that $\mathbb{P}\left(\tau_{\mathcal{E}}=\cdot\right)$ exists only if $\tau_{\mathcal{E}}<+\infty, \mathbb{P}-$ a.s. The latter implies that "the first event $\mathcal{E}$ must occur in a finite time $k, k>k_{p}$, almost surely or with probability 1". This requirement in practice can be understood as "the event $\mathcal{E}$ must occur at some future time". If this condition does not hold, $\mathbb{P}\left(\tau_{\mathcal{E}}=\cdot\right)$ could still exist, but without fulfilling the axiom of probability $\sum_{k \in \mathbb{N}} \mathbb{P}\left(\tau_{\mathcal{E}}=k\right)=1$.

\subsection{Application to Fatigue Crack Prognosis}

The theoretical contributions presented in Section 2 include their corresponding mathematical demonstrations and thus, in our humble opinion, do not need further validation. Nonetheless, in this section the authors intend to illustrate how these abstract mathematical statements can be used to solve a practical engineering application: the characterization of Time-of-Failure probability distributions in the context of failure prognostics problem; and more specifically, the problem of fatigue crack growth prognosis. For this purpose, a simplified stochastic degradation model is used to describe the growth of a fatigue crack in a test coupon as a function of loading cycles. The event $\mathcal{E}$ of interest corresponds to critical failures that may occur in mechanical systems with components that undergo fatigue crack processes, though it may not be clear that a specific crack lengths could trigger these events. The problem is addressed using the concept of uncertain event and is compared to the case of classical threshold-crossing-based events (i.e., critical failure always occurs when the crack length exceeds a known specific value). In all these cases, probability distributions for the first time of occurrence of the event are shown so as to develop a further discussion.

\subsubsection{Crack Growth Model}

In order to illustrate both the problem and the implementation of the concept of uncertain events, we have chosen to use the simplified discrete-time crack 


\subsubsection{Uncertain Event Definition}

As stated in Definition 1, and assuming the existence of a probability density that characterizes the crack length at each cycle given by the model described in Eq. (78), the statistics of an uncertain event $\mathcal{E}$ (in this case, critical failures in mechanical systems with components that undergo fatigue crack processes) are determined by the definition of $\mathbb{P}\left(E_{k}=\mathcal{E} \mid x\right)$ (see Eq. (8)), which describes how likely is that event $\mathcal{E}$ occurs at a particular time instant $k$ given that the crack length is $x$. Without loss of generality, let us assume that the critical failure events of interest can be associated with crack lengths of approximately 
$\bar{x}=100$. Thus, we may define the uncertain event likelihood function

$$
\mathbb{P}\left(E_{k}=\mathcal{E} \mid x\right)=\frac{1}{1+e^{-\alpha(x-\bar{x})}}, \alpha>0, \forall k \in \mathbb{N} .
$$

to provide a characterization of the uncertainty related to the occurrence of critical failure events in terms of the condition of the test coupon. Moreover, by using this critical failure likelihood, it is still possible to go back to a thresholdbased failure characterization (see Remark 2 ) by simply studying the limit

$$
\lim _{\alpha \rightarrow+\infty} \frac{1}{1+e^{-\alpha(x-\bar{x})}}=\mathbb{1}_{\{x \in \mathbb{R}: x>\bar{x}\}}(x) .
$$

\section{Remark 4. [Example of time-invariant uncertain event likelihood func- tion] \\ Eq. (79) corresponds to an example of a time-invariant uncertain event likeli- hood function $\mathbb{P}\left(E_{k}=\mathcal{E} \mid x\right)$ (see Remark 1 ).}

\section{Remark 5. [How to define the uncertain event likelihood function?]}

The uncertain event likelihood function that characterizes the uncertainty of the failure event can be built either using post-mortem statistical analysis or expert knowledge. The post-mortem statistical analysis requires the availability of run-to-failure data that could be used to reconstruct the trajectory of system condition indicators prior to the failure event. Values of condition indicators at the recorded failure events can be used to build a non-parametric likelihood function (in other words, to build an empirical joint probability mass function for condition indicators at the moment in which the system failed). Alternatively, it is always possible to adjust the parameters of a known function to fit the data. The use of expert knowledge would give rise to an epistemic source of uncertainty, where the uncertain event likelihood function is adjusted according to an expert's criteria. Bayesian approaches can always be used to fuse prior expert knowledge with scarce run-to-failure data.

It is important to note that the shortcomings associated with these procedures are equivalent to those that one would face when trying to establish a threshold for the failure indicator. The introduction of uncertain event prognosis, however, 
provides a solid theoretical framework where the concept of uncertain event is properly recognized and characterized. Both researchers and practitioners can use this theoretical framework to safely explore different methods to define these likelihood functions according to the specific challenges they are facing.

\subsubsection{Method of Monte Carlo Simulations}

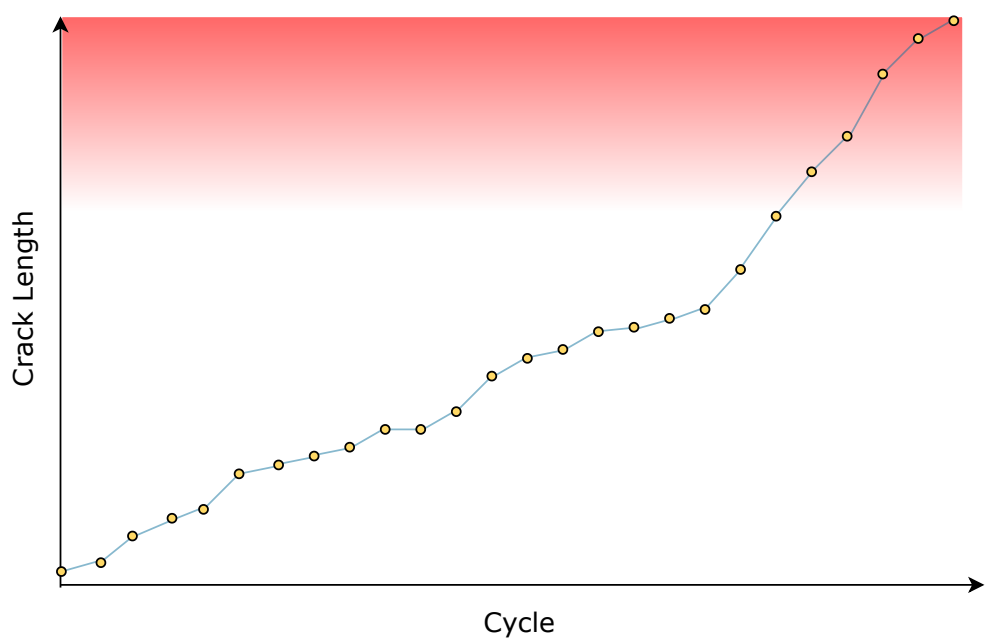

Figure 2: Single realization of the discrete-time stochastic process associated to crack length growth. The red color illustrates the magnitude of the uncertain event likelihood function $\mathbb{P}\left(E_{k}=\mathcal{E} \mid x\right)$; the greater the opacity, the greater the likelihood (see Definition 1 1 . In contrast, the classical approach to first event time prediction would not had shown a color hue, but an abrupt and discontinuous change from color white to red.

The notion of uncertain event incorporates a new degree of freedom for uncertainty characterization. In order to show how this new uncertainty source may impact event predictions, hereby we study its effect on the probability distribution associated to $\tau_{\mathcal{E}}$. Monte Carlo simulations are employed below to perform the required computation given their capacity to calculate expectations with arbitrary accuracy by simply increasing the number of simulations, denoted as $N \in \mathbb{N}$. Besides, let $x_{k_{p}+1: k}^{(i)}=\left\{x_{j}^{(i)}\right\}_{j=k_{p}+1}^{k}$ denote the $i$-th realization of the stochastic process simulated from cycle $k_{p}$ up to cycle $k$ (see Fig. 2), described by the crack growth model (Markov process) of Eq. (78), with $i \in\{1, \ldots, N\}$, 
$N>>1$. According to Theorem 1, the probability $\mathbb{P}\left(\tau_{\mathcal{E}}=k\right)$, with $\tau_{\mathcal{E}}=\tau_{\mathcal{E}}\left(k_{p}\right)$, can be approximated as:

$$
\begin{aligned}
& \mathbb{P}\left(\tau_{\mathcal{E}}=k\right)=\int_{\mathbb{X}_{k_{p}+1: k}} \mathbb{P}\left(E_{k}=\mathcal{E} \mid x_{k}\right) \prod_{j=k_{p}+1}^{k-1}\left(1-\mathbb{P}\left(E_{j}=\mathcal{E} \mid x_{j}\right)\right) d F_{X_{k_{p}+1: k}}\left(x_{k_{p}+1: k}\right) \\
& =\int_{\mathbb{X}_{k_{p}+1: k}} \mathbb{P}\left(E_{k}=\mathcal{E} \mid x_{k}\right) \prod_{j=k_{p}+1}^{k-1}\left(1-\mathbb{P}\left(E_{j}=\mathcal{E} \mid x_{j}\right)\right) p\left(x_{k_{p}+1: k}\right) d x_{k_{p}+1: k} \\
& \approx \int_{\mathbb{X}_{k_{p}+1: k}} \mathbb{P}\left(E_{k}=\mathcal{E} \mid x_{k}\right) \prod_{j=k_{p}+1}^{k-1}\left(1-\mathbb{P}\left(E_{j}=\mathcal{E} \mid x_{j}\right)\right)\left(\frac{1}{N} \sum_{i=1}^{N} \delta_{x_{k_{p}+1: k}^{(i)}}\left(x_{k_{p}+1: k}\right)\right) d x_{k_{p}+1: k} \\
& =\frac{1}{N} \sum_{i=1}^{N} \mathbb{P}\left(E_{k}=\mathcal{E} \mid x_{k}^{(i)}\right) \prod_{j=k_{p}+1}^{k-1}\left(1-\mathbb{P}\left(E_{k}=\mathcal{E} \mid x_{j}^{(i)}\right)\right)
\end{aligned}
$$

As depicted in Fig. 2, each realization of the stochastic process describing the evolution of the crack growth throughout usage cycles (the $i$-th for example), determines a likelihood for the occurrence of the uncertain event (material futility in this case). In this example, the figure illustrates the magnitude of the uncertain event likelihood function of Eq. (8) in terms of an hue over the crack length space with colors that go from a clear white to red progressively. The smoothness of this changing color depends, in this case, of the parameter $\alpha$ included in Eq. (8). In order to study the impact of this parameter on the probability mass distribution $\mathbb{P}\left(\tau_{\mathcal{E}}=\cdot\right)$, we explore different values, which are shown in Table 2 ,

\begin{tabular}{|c|c|c|c|c|c|}
\multicolumn{1}{c|}{} & $\alpha_{1}$ & $\alpha_{2}$ & $\alpha_{3}$ & $\alpha_{4}$ & $\alpha_{+\infty}$ \\
\hline Values & 0.1 & 0.3 & 1.0 & 3.3 & $\alpha \rightarrow+\infty$ \\
\hline
\end{tabular}

Table 2: Values considered for the parameter $\alpha$ in the definition of the uncertain event likelihood function $\mathbb{P}\left(E_{k}=\mathcal{E} \mid x\right)$ shown in Eq. 79 .

For clarity purposes, Fig. 3 illustrates how the uncertain event likelihood function looks like for the aforementioned values for the parameter $\alpha$. A special 

$\alpha \rightarrow+\infty$ (see Eq. (80)).

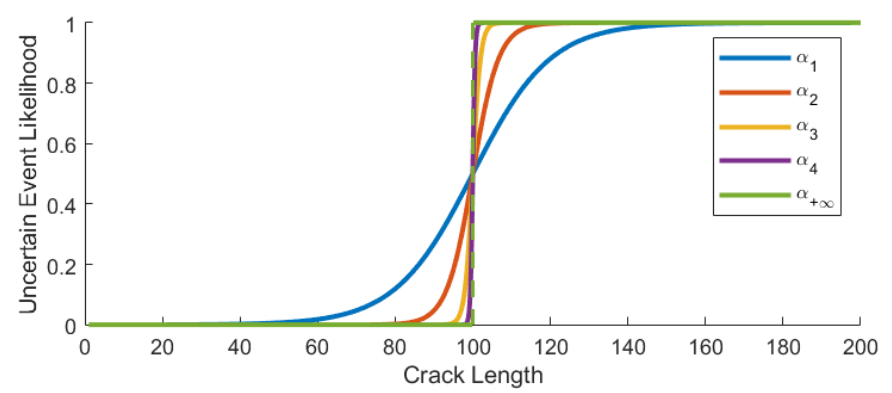

Figure 3: Uncertain event likelihood $\mathbb{P}\left(E_{k}=\mathcal{E} \mid x\right)$ as a function of the crack length $x \in \mathbb{R}_{+}$ for different values of the parameter $\alpha$ in Eq. 79. The parameter value $\alpha_{+\infty}$ depicts the behaviour of the function when $\alpha \rightarrow+\infty$ (see Eq. 80 ). The less the value of the parameter $\alpha$, the higher the uncertainty about an specific crack length depicting material futility. 
Remark 6. [Simulation of $\tau_{\mathcal{E}}$ by definition] Researchers within the PHM community often compute the probability distribution of $\tau_{\mathcal{E}}$ by definition, using for this purpose Monte Carlo simulations. Assuming that system failure can be characterized using a deterministic threshold (in other words, assuming $\alpha_{+\infty}$ for this particular case study), this methodology would be equivalent to simulate $N$ (with $N$ being a natural number large enough to reach convergence) possible future trajectories of the system state to characterize the probability of failure. Indeed, considering the definition of $\tau_{\mathcal{E}}$ (see Definition 2), an histogram of failure times can be made by simply counting the number of times that the simulated system state trajectories hit the failure threshold for the first time. There is a consensus among members of the PHM community regarding the fact that such histogram converges to the probability distribution of $\tau_{\mathcal{E}}$ when $N \rightarrow+\infty$. With this in mind, it is possible to notice that Eq. (84) describes exactly this procedure when the uncertain event likelihood function $\mathbb{P}\left(E_{k}=\mathcal{E} \mid x\right)$ describes a failure threshold (i.e. when it is an indicator function), as explained in Remark 2. In this regard, the semi-closed analytical expressions for $\mathbb{P}\left(\tau_{\mathcal{E}}=\cdot\right)$ provided in Theorems 1 and 2 of this article formalize this procedure analytically and furthermore, extend them to more general cases than a failure threshold, where there is uncertainty regarding how events are triggered.

\subsubsection{Simulation Results}

Let us consider that predictions begin at the cycle number $k_{p}=100$, at which an initial crack is detected and whose length is negligible (considered as $x_{k_{p}}=e^{-10}$ for simulations) and, additionally, a cycle number $k_{h}=1000$ at which simulations are stopped. Fig. 4 shows an example of how it would look like to simulate one hundred random crack growth trajectories. However, the Monte Carlo method described in Section 3.2.3 to approximate $\mathbb{P}\left(\tau_{\mathcal{E}}=k\right)$ with $k \in \mathbb{N}$ requires the amount of simulations to be such that $N \rightarrow+\infty$, which is not feasible in practice, but good approximations can be obtained when $N$ is a "sufficiently large" (where "sufficiently large" depends on dimension of the state vector, uncertainty sources, complexity of the model, among others). In 


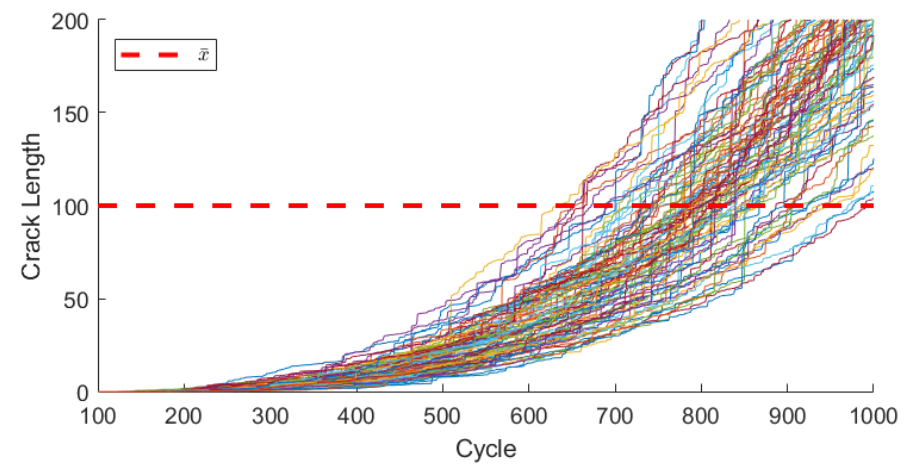

Figure 4: Example of 100 realizations of the crack growth model. The dashed horizontal line depicts a crack length of around $\bar{x}=100$ at which the material would become useless, though there is uncertainty about it (see Section 3.2 .2 .

this regard, the results of performing a total amount of $N=10^{7}$ Monte Carlo simulations for each of the $\alpha$ parameters explained in Section 3.2 .3 are shown in Table 3 and Fig. 5. Higher values of $N$ were discarded as they produce negligible effects on the results.

\begin{tabular}{|c|c|c|c|c|c|}
\cline { 2 - 6 } \multicolumn{1}{c|}{} & $\alpha_{1}$ & $\alpha_{2}$ & $\alpha_{3}$ & $\alpha_{4}$ & $\alpha_{+\infty}$ \\
\hline $\mathbb{E}\left\{\tau_{\mathcal{E}}\right\}$ & 660.8835 & 766.3128 & 783.6094 & 786.7342 & 787.4333 \\
\hline $\mathbb{S} t d\left\{\tau_{\mathcal{E}}\right\}$ & 102.6699 & 82.0342 & 82.7552 & 82.9145 & 82.9521 \\
\hline$\sum_{k=k_{p}}^{k_{h}} \mathbb{P}\left(\tau_{\mathcal{E}}=k\right)$ & 1.0000 & 0.9988 & 0.9970 & 0.9964 & 0.9962 \\
\hline
\end{tabular}

Table 3: Results in terms of expected values, standard deviations and probability mass within a cycle span between $k_{p}$ and $k_{h}$. The information is provided for each of the values considered for the parameter $\alpha$ in the definition of the uncertain event likelihood $\mathbb{P}\left(E_{k}=\mathcal{E} \mid x\right)$ of Eq. 79, which are shown in Table 2

The probability distributions for $\tau_{\mathcal{E}}$ depicted in Fig. 5 are quite illustrative regarding how uncertainty on the relationship between actual crack lengths and the occurrence of critical failures may be expressed in terms of $\tau_{\mathcal{E}}$ statistics. As the shape of the failure probability distributions is similar to a Gaussian bell, the expected values and standard deviations presented in Table 3 condense 


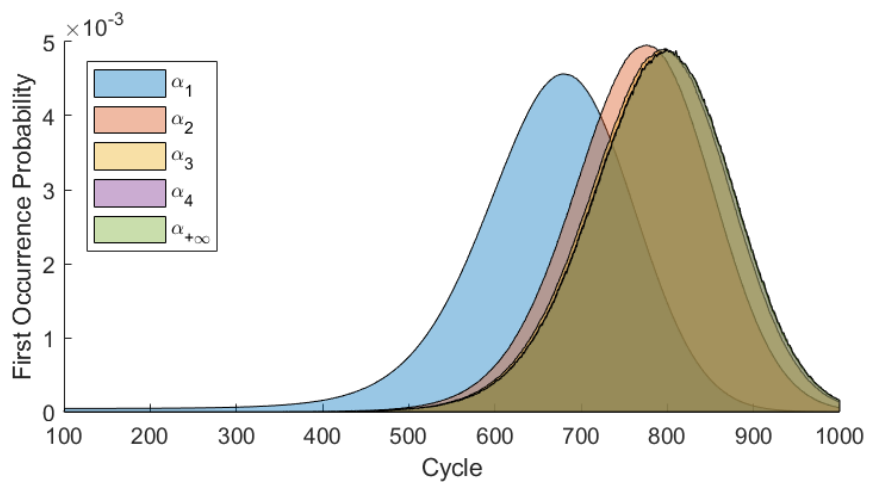

Figure 5: Computation of time probability distributions for the first occurrence of uncertain future events under different definitions of uncertain event likelihood function $\mathbb{P}\left(E_{k}=\mathcal{E} \mid x\right)$, which varies according to the different values of the parameter $\alpha$ (see Eq. 779) shown in Table 2 The parameter value $\alpha_{+\infty}$ depicts the behaviour of the function when $\alpha \rightarrow+\infty$ (see Eq. 80 ). The less the value of the parameter $\alpha$, the higher the uncertainty about the cycle at which critical cracks could lead to critical failures.

roughly all their information required to properly analyze the results.

The current standard approach of threshold crossing found in the literature is exactly represented by $\alpha_{+\infty}$. By taking it as point of comparison, it is straightforward to note from the expected values that, as $\alpha$ decreases, the probability distributions of $\tau_{\mathcal{E}}$ are shifted to left. In parallel, the standard deviations increase, spreading probabilities over a wider cycle span. This behaviour is naturally produced by any uncertainty source suggesting probability of earlier events. Indeed, the definition of uncertain event likelihood function (see Section 3.2.2 suggests that critical failures in mechanical components are likely to occur for crack lengths lower to $\bar{x}$, which is considered as threshold in the case of $\alpha_{+\infty}$. This means that it is probably to experience critical failures in a smaller amount of loading cycles, which explains the behaviour of the expected values in Table 3 The standard deviations, on the other hand, are obtained just as an outcome of incorporating a new uncertainty source in the study. Finally, the similar results obtained with $\alpha_{3}, \alpha_{4}$ and $\alpha_{+\infty}$ are consistent with the fact that their uncertain event likelihood functions are strongly similar as well, as shown 
in Fig. 3

\section{Conclusion}

For more than fifty years, researchers from several disciplines have approached the problem of predicting the time of occurrence of events in the future. For this reason, they have explored this idea assuming a wide variety of types of stochastic process. However, the common approach has always been to trigger an event once a particular threshold or specific zone in a higher dimensional space, is reached. The underlying reason is mainly based on an aiming at achieving closed-form mathematical expressions. In this regard, uncertainty on this threshold or higher dimensional zone has been addressed just for a reduced quantity of stochastic processes.

In this paper it has been introduced a new notion of uncertain event that generalizes the standard way of event definition for predicting its first time of occurrence in the future. Although this idea is not new, one of the greatest contributions presented in this paper is the formalization of this concept throughout a rigorous approach from Probability Theory. Moreover, the concept of hazard zone known in the discipline of Prognostics and Health Management has finally got formalized as well. On the other hand, the second -and no less importantcontribution is to show its straightforward applicability with a simple example of fatigue crack growth, where practical guidelines and implications of the new concepts introduced have been provided and discussed.

\section{Acknowledgment}

This work has been supported by FONDECYT Chile Grant Nr. 1170044, and the Advanced Center for Electrical and Electronic Engineering, AC3E, Basal Project FB0008, ANID. Also, the authors want to thank project ANID PIA ACT1405 and ANID-PFCHA/Doctorado Nacional/2017-21171097 for supporting David E. Acuña-Ureta doctoral studies. 


\section{References}

[1] S. Redner, A guide to first-passage processes, Cambridge University Press, Cambridge, 2001.

[2] R. Metzler, G. Oshanin, , S. Redner, First-passage phenomena and their applications, World Scientific, Singapore, 2014.

[3] J. Drugowitsch, Fast and accurate Monte Carlo sampling of first-passage times from Wiener diffusion models, Scientific Reports 6.

[4] S. Herrmann, C. Zucca, Exact simulation of the first-passage time of diffusions, arXiv e-prints (2017) arXiv:1705.06881.

[5] M.-L. T. Lee, G. A. Whitmore, Threshold regression for survival analysis: Modeling event times by a stochastic process reaching a boundary, Statistical Science 21 (4) (2006) 501-513.

[6] A. Lipton, V. Kaushansky, On the first hitting time density of an OrnsteinUhlenbeck process, arXiv e-prints (2018) arXiv:1810.02390.

[7] T. Lancaster, A stochastic model for the duration of a strike, J. Roy. Statist. Soc. Ser. A 135 (2) (1972) 257-271.

[8] G. A. Whitmore, First-passage-time models for duration data: Regression structures and competing risks, Journal of the Royal Statistical Society. Series D (The Statistician) 35 (2) (1986) 207-219.

[9] M. A. Muñoz, G. Grinstein, Y. Tu, Survival probability and field theory in systems with absorbing states, Phys. Rev. E 56 (5) (1997) 5101-5105.

[10] L. V. Ballestra, G. Pacelli, Computing the survival probability density function in jump-diffusion models: A new approach based on radial basis functions, Engineering Analysis with Boundary Elements 35 (9) (2011) $1075-1084$. 
[11] N. Levernier, M. Dolgushev, O. Bénichou, R. Voituriez, T. Guérin, Survival probability of stochastic processes beyond persistence exponents, Nature Communications 10 (2990).

[12] M. G. Pecht, M. Kang, Prognostics and Health Management of Electronics: Fundamentals, Machine Learning, and the Internet of Things, Wiley-IEEE Press, 2018.

[13] K. Goebel, M. Daigle, A. Saxena, S. Sankararaman, I. Roychoudhury, J. Celaya, Prognostics: The Science of Making Predictions, 2017.

[14] G. Vachtsevanos, F. L. Lewis, M. Roemer, A. Hess, B. Wu, Intelligent Fault Diagnosis and Prognosis for Engineering Systems, Wiley, 2006.

[15] A. Szabo, K. Schulten, Z. Schulten, First passage time approach to diffusion controlled reactions, The Journal of Chemical Physics 72 (8) (1980) 43504357.

[16] M. Razbin, P. Benetatos, A. A. Moosavi-Movahedi, A first-passage approach to the thermal breakage of a discrete one-dimensional chain, Soft Matter 15 (2019) 2469-2478.

[17] M. Nyberg, T. Ambjörnsson, L. Lizana, A simple method to calculate first-passage time densities with arbitrary initial conditions, New Journal of Physics 18.

[18] S. Ahmad, I. Nayak, A. Bansal, A. Nandi, D. Das, First passage of a particle in a potential under stochastic resetting: A vanishing transition of optimal resetting rate, Phys. Rev. E 99 (2) (2019) 022130.

[19] Z. Hu, L. Cheng, B. J. Berne1, First passage time distribution in stochastic processes with moving and static absorbing boundaries with application to biological rupture experiments, The Journal of Chemical Physics 133 (3).

[20] T. Chou, M. R. D'Orsogna, First passage problems in biology, arXiv eprints (2014) arXiv:1408.4518. 
[21] H. C. Tuckwell, Introduction to theoretical neurobiology. Volume 2: Nonlinear and Stochastic Theories, Cambridge University Press, Cambridge, 1988.

[22] D. Holcman, A. Marchewka, Z. Schuss, Survival probability of diffusion with trapping in cellular neurobiology, Phys. Rev. E 72 (3).

[23] A. L. Lloyd, R. M. May, How viruses spread among computers and people, Science 292 (5520) (2011) 1316-1317.

[24] D. J. Navarro, I. G. Fuss, Fast and accurate calculations for first-passage times in Wiener diffusion models, Journal of Mathematical Psychology 53 (4) (2009) 967-980.

[25] G. Bakshi, G. Panayotov, First-passage probability, jump models, and intra-horizon risk, Journal of Financial Economics 95 (2010) 20-40.

[26] J. Janssen, O. Manca, R. Manca, Applied Diffusion Processes from Engineering to Finance, Wiley-ISTE, Hoboken, 2013.

[27] M. Jaskowski, D. van Dijk, First-passage-time in discrete time, Research Seminar, Erasmus School of Economics (ESE), Erasmus University Rotterdam.

[28] J. H. Abbring, T. Salimans, The likelihood of mixed hitting times, arXiv e-prints (2019) arXiv:1905.03463.

[29] Y. S. Sherif, M. L. Smith, First-passage time distribution of Brownian motion as a reliability model, IEEE Transactions on Reliability R-29 (5) (1980) 425-426.

[30] V. Pieper, M. Dominé, P. Kurth, Level crossing problems and drift reliability, Mathematical Methods of Operations Research 45 (3) (1997) 347-354.

[31] V. Srivastava, S. F. Feng, J. D. Cohen, N. Ehrich Leonard, A. Shenhav, A martingale analysis of first passage times of time-dependent Wiener diffusion models, Journal of Mathematical Psychology 77 (2017) 94-110. 
[32] O. Artime, N. Khalil, R. Toral, M. San Miguel, First-passage distributions for the one-dimensional Fokker-Planck equation, Phys. Rev. E 98 (4).

[33] C. Buchera, M. Di Paola, Efficient solution of the first passage problem by path integration for normal and Poissonian white noise, Probabilistic Engineering Mechanics 41 (2015) 121-128.

[34] W. Li, W. Xu, J. Zhao, Y. Jin, First-passage problem for strong nonlinear stochastic dynamical systems, Chaos, Solitons \& Fractals 28 (2) (2006) 414-421.

[35] M. J. Kearney, S. N. Majumdar, On the area under a continuous time Brownian motion till its first-passage time, Journal of Physics A: Mathematical and General 38 (19).

[36] H. Li, M. Shaked, On the first passage times for Markov processes with monotone convex transition kernels, Stochastic Processes and their Applications 58 (2) (1995) 205-216.

[37] J. B. Roberts, First-passage probabilities for randomly excited systems: Diffusion methods, Probabilistic Engineering Mechanics 1 (2) (1986) 6681.

[38] M. Burnashev, A. Tchamkerten, Estimating a random walk first-passage time from noisy or delayed observations, IEEE Transactions on Information Theory 58 (7) (2012) 4230-4243.

[39] S. N. Majumdar, Universal first-passage properties of discrete-time random walks and Lévy flights on a line: Statistics of the global maximum and records, Physica A: Statistical Mechanics and its Applications 389 (20) (2010) 4299-4316.

[40] H. Li, M. Shaked, Ageing first-passage times of Markov processes: A matrix approach, Journal of Applied Probability 34 (1) (1997) 1-13. 
[41] T. Koski, B. Jung, G. Högnäs, Exit times for ARMA processes, Advances in Applied Probability 50 (A) (2018) 191-195.

[42] C. Baumgarten, Survival probabilities of autoregressive processes, ESAIM: Probability and Statistics 18 (2014) 145-170.

[43] B. Jung, Exit times for multivariate autoregressive processes, Stochastic Processes and their Applications 123 (8) (2013) 3052-3063.

[44] X. Guo, A. H. del Valle, O. Hernández-Lerma, First passage problems for nonstationary discrete-time stochastic control systems, European Journal of Control 18 (6) (2012) 528-538.

[45] E. Di Nardo, On the first passage time for autoregressive processes, Scientiae Mathematicae Japonicae 67 (2010) 137-152.

[46] A. Novikov, N. Kordzakhia, Martingales and first passage times of AR(1) sequences, Stochastics: An International Journal of Probability and Stochastic Processes 80 (2-3) (2008) 197-210.

[47] H. Larralde, A first passage time distribution for a discrete version of the Ornstein-Uhlenbeck process, Journal of Physics A: Mathematical and General $37(12)$.

[48] G. K. Basak, K.-W. R. Ho, Level-crossing probabilities and first-passage times for linear processes, Advances in Applied Probability 36 (2) (2004) 643-666.

[49] L. M. Ricciardi, S. Sato, First-passage-time density and moments of the Ornstein-Uhlenbeck process, Journal of Applied Probability 25 (1) (1988) $43-57$.

[50] V. Linetsky, Computing hitting time densities for CIR and OU diffusions: applications to mean-reverting models, Journal of Computational Finance 7 (4) (2004) 1-22. 
[51] L. Alili, P. Patie, J. L. Pedersen, Representations of the first hitting time density of an Ornstein-Uhlenbeck process, Stochastic Models 21 (4) (2005) 967-980.

[52] H. R. Lerche, Boundary crossing of Brownian motion, Lecture Notes in Statistics 40.

[53] S. Ghazizadeh, M. Barbato, E. Tubaldi, New analytical solution of the firstpassage reliability problem for linear oscillators, Journal of Engineering Mechanics 138 (6) (2012) 695-706.

[54] J. Song, A. D. Kiureghian, Joint first-passage probability and reliability of systems under stochastic excitation, Journal of Engineering Mechanics 132 (1) (2006) 65-77.

[55] S. K. Au, J. L. Beck, First excursion probabilities for linear systems by very efficient importance sampling, Probabilistic Engineering Mechanics 16 (3) (2001) 193-207.

[56] A. Naess, Crossing rate statistics quadratic transformations of Gaussian processes, Probabilistic Engineering Mechanics 16 (3) (2001) 209-217.

[57] E. Di Nardo, A. G. Nobile, E. Pirozzi, L. M. Ricciardi, A computational approach to first-passage-time problems for Gauss-Markov processes, Advances in Applied Probability 33 (2) (2001) 453-482.

[58] V. Bayer, C. Bucher, Importance sampling for the first passage problems of nonlinear structures, Probabilistic Engineering Mechanics 14 (1-2) (1999) $27-32$.

[59] S. Engelund, R. Rackwitz, C. Lange, Approximations of first-passage times for differentiable processes based on higher-order threshold crossings, Probabilistic Engineering Mechanics 10 (1) (1995) 53-60.

[60] R. S. Langley, A first passage approximation for normal stationary random processes, Journal of Sound and Vibration 122 (2) (1988) 261-275. 
[61] P. H. Madsen, S. Krenk, An integral equation method for the first-passage problem in random vibration, J. Appl. Mech. 51 (3) (1984) 674-679.

[62] P. Hänggi, P. Talkner, Non-Markov processes: The problem of the mean first passage time, Zeitschrift für Physik B Condensed Matter 45 (1) (1981) 79-83.

[63] S. H. Crandall, First-cross probabilities of the linear oscillator, Journal of Sound and Vibration 12 (3) (1970) 285-299.

[64] J. J. Coleman, Reliability of aircraft structures in resisting chance failure, Probabilistic Engineering Mechanics 7 (5) (1959) 639-645.

[65] D. A. Darling, A. J. F. Siegert, The first passage problem for a continuous Markov process, The Annals of Mathematical Statistics 24 (4) (1953) 624639.

[66] B. Sildnes, B. H. Lindqvist, Modeling of semi-competing risks by means of first passage times of a stochastic process, Lifetime Data Analysis 24 (1) (2018) 153-175.

[67] Y. Shu, Q. Feng, D. W. Coit, Life distribution analysis based on Lévy subordinators for degradation with random jumps, Naval Research Logistics (NRL) 62 (6) (2015) 483-492.

[68] C. Paroissin, A. Salami, Failure time of non homogeneous Gamma process, Communications in Statistics - Theory and Methods 43 (15) (2014) 31483161.

[69] A. Lehmann, Joint modeling of degradation and failure time data, Journal of Statistical Planning and Inference 139 (5) (2009) 1693-1706.

[70] Y. Yang, G.-A. Klutke, Lifetime-characteristics and inspection-schemes for Lévy degradation processes, IEEE Transactions on Reliability 49 (4) (2000) 377-382. 
[71] M. Abdel-Hameed, Life distribution properties of devices subject to a Lévy wear process, Mathematics of Operations Research 9 (4) (1984) 479-634.

[72] E. Lughofer, M. Sayed-Mouchaweh (Eds.), Predictive Maintenance in Dynamic Systems: Advanced Methods, Decision Support Tools and RealWorld Applications, Springer, Cham, 2019.

[73] M. Orchard, G. Vachtsevanos, A particle-filtering approach for on-line fault diagnosis and failure prognosis, Trans. Inst. Meas. Control 200931 (2009) 221-246.

[74] D. E. Acuña, M. E. Orchard, A theoretically rigorous approach to failure prognosis, Annual Conference of the Prognostics and Health Management Society 201810 (1).

[75] D. E. Acuña, M. E. Orchard, Particle-filtering-based failure prognosis via sigma-points: Application to lithium-ion battery state-of-charge monitoring, Mech. Syst. Signal Process. 85 (2017) 827-848.

[76] D. E. Acuña, R. J. Saona, M. E. Orchard, Conditional predictive Bayesian Cramér-Rao lower bounds for prognostic algorithms design, Applied Soft Computing 72 (2018) 647-665.

[77] M. Daigle, K. Goebel, Model-based prognostics with concurrent damage progression processes, IEEE Transactions on Systems, Man, and Cybernetics: Systems 43 (4) (2013) 535-546.

[78] M. Daigle, S. Sankararaman, Advanced methods for determining prediction uncertainty in model-based prognostics with application to planetary rovers, Annual Conference of the Prognostics and Health Management Society 2013.

[79] A. A. Taflanidis, J. L. Beck, An efficient framework for optimal robust stochastic system design using stochastic simulation, Computer Methods in Applied Mechanics and Engineering 198 (1) (2008) 88-101. 
[80] A. A. Taflanidis, J. L. Beck, Stochastic subset optimization for optimal reliability problems, Probabilistic Engineering Mechanics 23 (2-3) (2008) 324-338.

[81] M. A. Valdebenito, G. I. Schuëller, A survey on approaches for reliabilitybased optimization, Structural and Multidisciplinary Optimization 42 (5) (2010) 645-663.

[82] I. Papaioannou, K. Breitung, D. Straub, Reliability sensitivity estimation with sequential importance sampling, Structural Safety 75 (2018) 24-34.

[83] F. Cadini, E. Zio, D. Avram, Monte Carlo-based filtering for fatigue crack growth estimation, Probabilistic Engineering Mechanics 24 (3) (2009) 367373. 